\title{
Evolution of Adaptive Population Control in Multi-agent Systems
}

\author{
Benjamin E. Beckmann and Philip K. McKinley \\ Department of Computer Science and Engineering \\ Michigan State University \\ East Lansing, Michigan 48824 \\ E-mail: \{beckma24, mckinley\} @ cse.msu.edu
}

\begin{abstract}
Dynamic population management is an important aspect of multi-agent systems. In artificial immune systems, for example, a shortage of agents can lead to undetected threats, while an overabundance of agents can degrade quality of service and if unchecked, even create new vulnerabilities. Unfortunately, designing an effective strategy for population management is complicated by the myriad of possible circumstances and environmental conditions the agents may face after deployment. In this paper, we present the results of a study in applying digital evolution to the population management problem. In digital evolution, populations of self-replicating computer programs evolve in a user-defined computational environment, where they are subject to mutations and natural selection. Our results demonstrate that populations of digital organisms are capable of evolving self-adaptive replication behaviors that respond to attack fluctuations, as well as clever strategies for cooperating to mitigate attacks. This study provides evidence that digital evolution may be a useful tool in the design of selforganizing and self-adaptive agent-based systems.
\end{abstract}

Keywords: digital evolution, cooperative behavior, natural selection, population regulation, multi-agent system.

\section{Introduction}

The level of cooperation in nature is remarkable and can be observed within and across many scales: sub-cellular, cellular, organ, organism, and ecosystem. Moreover, abnormalities in a subsystem at any scale can cause disastrous results. For example, if the number of white blood cells (or leukocytes) within a human's immune system is not effectively controlled, leukemia (too many leukocytes) or leukopenia (too few leukocytes) can develop, possibly resulting in death. Similarly, population management issues can present problems for an agent-based computational sys- tem. For example, if the number of detector agents in an artificial immune system (AIS) is not properly controlled, then the system's ability to detect a threat may degrade due to overconsumption of resources, leading to reduced quality of service (QoS) and possibly system failure [30]. Enforcing a global limit on the number of agents within a decentralized system is complicated by delays in communication and lack of synchronization, and may also reduce the ability of the system to respond quickly to a large-scale attack. Furthermore, agents may experience unforeseen circumstances and environmental conditions, exacerbating the problem of regulating a population. Therefore, an adaptive population control mechanism is appealing.

Much like a natural organism's immune system responds to antigens [23], an AIS is designed to protect a computational system from intrusion by unauthorized or disruptive entities. This biological analogy has been the focus of studies on computer immunology $[12,20]$ and is one of the catalysts for the field of autonomic computing [24]. In addition to designing systems by mimicking features of immune systems observed in nature (biomimetics), some approaches exploit the process of natural selection which, over millions of generations, constructed the mechanisms for detecting and eliminating pathogens within an organism. Indeed, several studies have used evolutionary computation methods such as the genetic algorithm [22] to assist in the design of individual AIS agents $[1,15]$.

In this paper we investigate the application of evolutionary computation to the design of a group-wide behavior, namely adaptive population control for autonomous mobile agents. Instead of using a genetic algorithm (GA), however, we explore the potential benefits of another form of evolutionary computation known as digital evolution (DE) [2]. In digital evolution, a population of self-replicating computer programs exists in a computational environment and is subject to mutations and natural selection. These "digital organisms" are provided with limited resources whose use must be carefully balanced if they are to survive. Since digital organisms interact with one another and are responsible 
for their own replication, the process is closer to natural evolution than traditional forms of evolutionary computation such as GAs. Designed primarily as a tool for evolutionary biologists, this "digital Petri dish" enables researchers to explore questions that are difficult or impossible to study with organic life forms $[3,14,28,35]$.

In addition to uses in biology, digital evolution provides a means to harness the power of natural selection and apply it to a variety of problems in designing computational systems. Digital evolution enables the designer to explore an enormous solution space, potentially discovering unintuitive algorithms that are robust under highly dynamic and adverse conditions. Our ongoing investigations explore the application of digital evolution to problems in distributed computing [31]. Many similarities can be drawn between the capabilities of a digital organism and an agent in a distributed system. Both are capable of replication, local computation, environmental interactions, and communication with other agents. In addition, these capabilities can be leveraged and coupled within a group to produce collaborative behaviors, i.e., swarms of agents, enabling the execution of complex group-level tasks through self-organization. In related studies we have demonstrated that populations of digital organisms can evolve the ability to perform collective data operations [7,25], elect leaders [26], forage for resources [8], and build semi-permanent structures [27].

The main contribution of this paper is to demonstrate a "proof of concept" that DE can produce a population of mobile agents exhibiting self-organization. Specifically, we focus on cooperative actions among neighboring organisms to mitigate attacks by changing their collective behavior in response to dynamic threat levels. This study also helps to identify evolutionary conditions that enable this global behavior to emerge in a population. Moreover, analyzing the genome of a particularly successful population provides insight into the complexity and effectiveness of solutions discovered through the evolutionary process. As with our other studies, algorithms for population management can be automatically translated into executable code and evaluated through either simulation or experimentation [8]. These activities constitute our ongoing work, discussed later.

The remainder of this paper is organized as follows. Section 2 presents related work on autonomous agents and population regulation. Section 3 includes a description of the DE system, Avida [32], used in this study. Section 4 contains a discussion of the methods used to promote the evolution of autonomous agents capable of self-regulating their population while maintaining an acceptable quality of service. Section 5 presents experimental results, followed by conclusions and future work in Section 6.

\section{Background}

As computing becomes more pervasive and decentralized, many design techniques (including autonomic agentbased systems [24]) have been proposed to accommodate the increasing complexity. In these systems, individual computational agents collaborate to perform a task based on administrative goals, and the system as a whole is selfmanaging. Therefore, after such a system is configured and initiated, little or no human interaction is required.

Autonomous agents have been applied to many different application areas in computer science [6,11,24], and the agent paradigm has been shown to be an effective approach to addressing dynamic reconfiguration, scalability, and self-protection. These advantages are derived from an agent's ability to act either individually or collaboratively, clone (or self-replicate) itself, and migrate across networks while maintaining state and performing tasks. When applied to the AIS domain, an agent's ability to be either active or passive depending on the state of the system is a major advantage. For example, when a system is not experiencing threats, agents can remain in a quiescent state, periodically waking and sensing the local environment. Once a threat is detected, the detecting agent can become active and send messages to other active agents, as well as propagate clones to neighboring nodes in order to localize, and possibly quell a threat [19].

Agent-based systems do, however, require population management to ensure the agents do not inhibit system functionality. For example, if agent lifetime is improperly configured, the system may produce unacceptable false positive or false negative detection rates [21]. In addition to an agent's lifetime, the number of agents in the system can also affect quality of service (QoS). If the number of detectors in an AIS is too small, then threats can go undetected, whereas if they are too numerous, the system can suffer from resource limitations. Adapting values associated with these management concerns (agent lifetime and number of agents) can directly affect a system's responsiveness, robustness, resiliency, and efficiency [11].

Population size regulation has been studied in genetic algorithm literature $[4,16,17]$. Generally, however the strategies used in those approaches are static and may require global knowledge. For example, in [17], the lifetime of an individual has a fixed maximum, and global knowledge is used to maintain sufficient diversity within a population. The DE method described here enables an agent to alter its life span, and does not use any global knowledge to promote diversity. While we do limit the maximum lifetime of an individual, this limit is extremely loose, greater than 50 times the maximum lifetime ever observed in our experiments.

Population management has also been addressed in systems-related fields [5, 13, 34]. Many approaches use 
a priori rules limiting population growth. For example, in [13] autonomous robots are evolved to capture a target while avoiding collisions. A predefined table specifies the replication rate, relative to the number of consecutively captured targets. In contrast, digital evolution involves no explicit control of the replication rate. Rather, natural selection drives a population to establish the number of individuals required to solve a problem. In addition, unlike the method described in [13], we do not allow information to be stored in the environment for use in stigmergic communication (e.g., digital pheromones). Our experiments show that even without these mechanisms, digital evolution is capable of producing agents that find and eliminate $90 \%$ of all system "attacks," while self-regulating the population size during low and high threat periods.

\section{Avida Digital Evolution Platform}

Avida is a well established artificial life platform used in evolutionary biology $[3,28,29,32]$ and more recently in distributed systems research [31].

Basic Architecture and Operation. In Avida, individuals, or digital organisms, compete for space within a fixedsize two-dimensional collection of cells. Each cell can contain at most one organism, which comprises a circular list of instructions (its genome) and a virtual CPU that executes those instructions, as shown at the top of Figure 1. Instructions perform simple arithmetic operations (addition, bitshift, increment, etc.), control execution flow, aid in selfreplication, enable the organism to move from one cell to another, and provide a means for the organism to interact with other organisms and the environment. An organism executes these instructions on its virtual CPU, which contains three general purpose registers (AX, BX, CX), two general purpose stacks, and special purpose heads which point to locations within the organism's genome. Similar to a traditional program counter and stack pointer, heads are used to control the flow of execution. The execution of an instruction costs both virtual CPU cycles and energy. Different instructions can be assigned different CPU cycle and energy costs.

Avida organisms are self replicating, that is, their genomes must contain instructions to create an offspring, which is placed in a randomly selected cell, terminating any previous inhabitant. Typically, an Avida population starts with a single seed organism capable only of replication. As organisms replicate, instruction-level mutations produce variation within the population. In this study, the ancestral organism contains 49 no-operation (NOP) instructions and a single REPRO instruction, which performs replication. The NOP instructions have no effect on the ancestral organism's observed behavior, or phenotype, excluding its gestation time. However, they do provide the evolutionary pro-

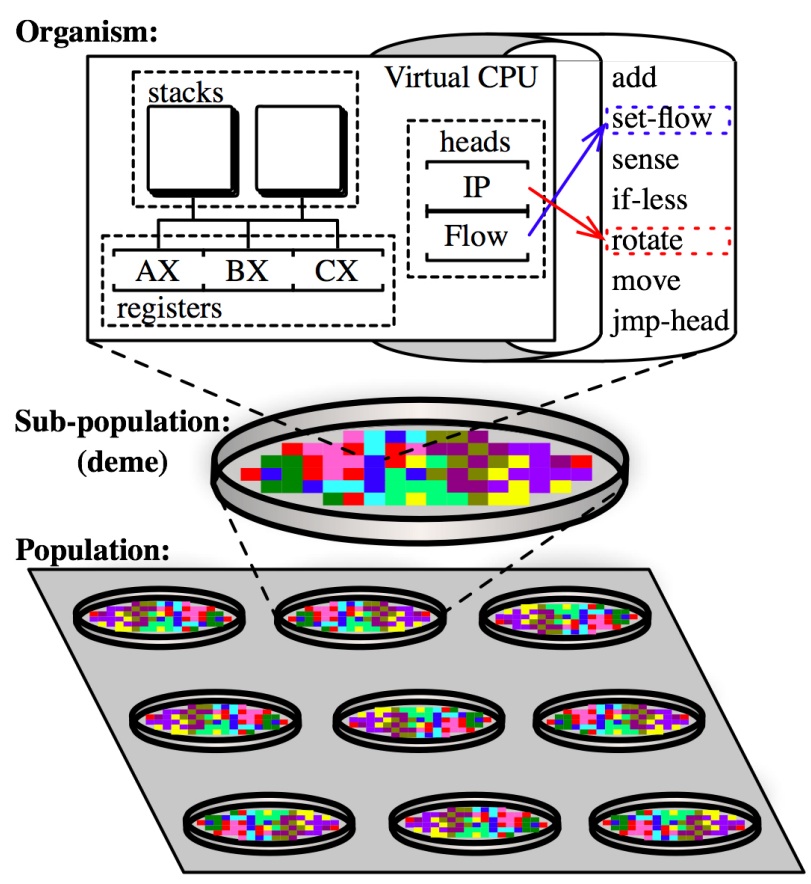

Figure 1. Population (bottom), sub-population (middle) and composition of a digital organism: genome (top right), virtual CPU (top left) with heads pointing to locations within the genome

cess more room to work, as mutations during replication bring different instructions into the genome.

Instead of an explicit fitness function, the competition for space drives selection in Avida: those organisms who replicate faster are more successful than those who do not. An organism's energy is used to calculate its metabolic rate using Equation 1. An organism's metabolic rate is inversely proportional to a user defined limit on the total number of instructions an organism can execute before its energy is depleted, assuming no new energy influx and all instructions cost 1 energy unit. Probabilistically, an organism with a higher metabolic rate will be given more virtual CPU cycles to execute instructions at a higher energy cost per instruction, calculated by Equation 2, than an organism with a lower metabolic rate. An organism can increase its energy, either individually or as part of a group, by exhibiting behaviors prescribed by the user.

$$
\begin{aligned}
& \text { metabolic rate }=\frac{\text { stored energy }}{\text { max. executed inst. }} \\
& \text { actual energy cost }= \text { metabolic rate } \times \\
& \text { inst. } \text { energy cost }
\end{aligned}
$$


In Avida, time is measured using an abstract unit called an update. During an update, an average organism is will probabilistically execute 30 instructions. When exploring a particular problem, we execute several batches of Avida runs, each with a different configuration, and analyze the evolutionary process and the resulting behaviors. A batch typically contains 20 runs, each of which starts with the same default organism. Since each run within a batch is started with a different random number seed, the populations take different evolutionary paths. Typically, each population evolves for at least 100,000 updates.

Demes and Multilevel Selection. In some studies we treat all organisms as part of a single population, in which case individual organisms compete against one another. However, in other cases, especially those involving the evolution of cooperative behavior, it is useful to subdivide the population and have groups of organisms compete. As shown at the bottom of Figure 1, a population of organisms can be subdivided into many sub-populations, called demes. All demes have identical environments and initial configurations. However, an organism within a deme can only interact with other organisms in the same deme. Subdividing the population this way is akin to constructing "multicellular" organisms, enabling the detection and eventual selection of demes that perform group-level behaviors.

Multilevel selection [36] can be described as the application of natural selection at different granularities. Avida supports user defined multilevel selection, specifically individual and deme-level selection. To enable deme-level selection, a deme is replicated when it satisfies a deme-level predicate, more generally thought of as a group-level behavior, such as flocking or consensus [26]. Upon deme replication, prior to creating an offspring deme, mutations are applied to the genome that was used to seed the parent deme. During this mutation process each instruction in the genome is subject to a $0.75 \%$ chance of being mutated to a random instruction. The newly created genome and its ancestral genomes make up the germline from which all seed organisms are produced. In contrast, other non-germline, or somatic, organisms play no role in deme replication. In addition to deme-level predicates, a deme's age is also used as a trigger for deme replication. This method allows for the bootstrapping of the evolutionary process by introducing mutations into a deme's germline. Figure 2 depicts the initial injection of the ancestral organism into every deme, and both age and predicate based deme replication methods.

While individual organisms within a deme are able to replicate, those replications do not involve mutations to the genome. Hence, all organisms within a deme are genetically identical. Floreano et.al. [18] have previously shown that this approach is effective in evolving cooperative behavior.

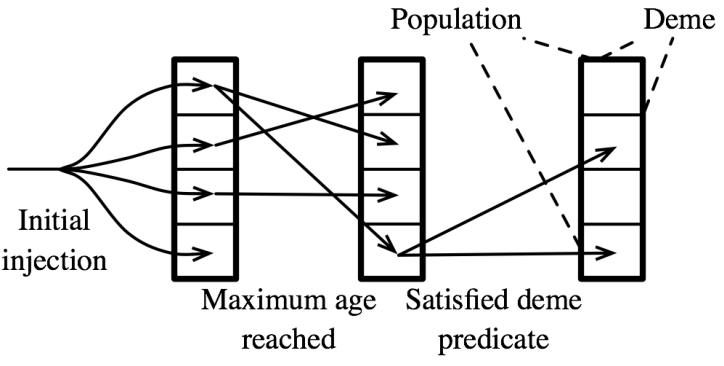

Figure 2. Example showing deme initialization and replication of germlines

\section{Evolving adaptive population control}

We investigated the evolution of an agent behavior to detect and mitigate "attacks" on cells in an energy efficient manner. We associated with each cell a certain amount of energy. When an organism enters a cell it acquires the energy within that cell and uses it to execute instructions. If an organism leaves the cell, its remaining energy is placed back into the cell and is unchanged until another organism enters the cell or the cell experiences an attack. An attack targets a single cell and acts as an energy sink. Attacks appear and are placed randomly in cells within a deme. When an attack is placed in a cell it draws down the energy within that cell, or an occupying organism, for the duration of the attack. (The duration and percentage of energy loss due to an attack are both fixed throughout a single run.) In the results discussed in Section 5, an attack's duration is set to 5 updates, and $1 \%$ of a cell's remaining energy is lost during every update during which the attack remains active. To conduct the study we added to Avida several new instructions related to communication, movement, environmental sensing, and changing their metabolism rate, as described below.

Active Messaging. First, we defined instructions for $a c$ tive messaging [33], enabling organisms to alter the execution of other organisms by sending messages to them. The active messaging functionality is provided through two instructions, SEND-ALARM-LOCAL (to all organisms in neighboring cells) and SEND-ALARM-GLOBAL (to all organisms in the deme). As with any other instruction, these enter the organisms' genomes through random mutations during replication, and evolution needs to put together sequences of instructions into meaningful behaviors. An alarm message can be in one of two states, low or high, determined by the contents of the BX register in the sending organism: if the value stored in the register is even, then a low-state alarm message is sent, otherwise a high-state alarm is sent. 
Both of these instructions cause each non-sleeping, receiving organism to immediately move its instruction pointer from its current position to the location of an alarm label instruction, if present. An alarm label is a special purpose no-operation instruction used solely as a target for such an alarm-induced jump. The state of an alarm message is used to determine which alarm label is targeted for a jump. We defined two alarm label instructions, ALARM-LABELLOW and ALARM-LABEL-HIGH, which can be jumped to depending on the state of the received alarm. When an organism receives an alarm its genome is searched in the forward direction, from the instruction pointer's current position, until the correct alarm label is found or the search fails. If a corresponding alarm label is found, the receiver organism's instruction pointer is set to that location. If no corresponding alarm label is found then no jump is performed.

Figure 3 depicts the execution of two neighboring organisms' genomes, initially organism $A$ (top) is about to execute a SEND-ALARM-MSG-LOCAL instruction, which will send a high-state alarm to organism $B$ (bottom). Once $A$ executes the instruction, organism $B$ 's execution jumps to the location of the ALARM-LABEL-HIGH instruction in its genome, skipping all instructions between the ROTATERIGHT and ALARM-LABEL-HIGH instructions. From this point on, both organisms execute their genomes sequentially until another alarm is received.

\begin{tabular}{|c|c|}
\hline \multicolumn{2}{|l|}{ Organism A (sender) } \\
\hline $\begin{array}{l}\text { ROTATE-RIGHT } \\
\ldots \\
\text { SEND-ALARM-MSG-LOCAL } \\
\text { MOVE } \\
\ldots \\
\text { ALARM-LABEL-HIGH High } \\
\text { REPRO }\end{array}$ & $\begin{array}{l}\text { ROTATE-RIGHT } \\
\ldots \\
\text { SEND-ALARM-MSG-LOCAL } \\
\text { MOVE } \\
\ldots \\
\text { ALARM-LABEL-HIGH } \\
\text { REPRO }\end{array}$ \\
\hline \multicolumn{2}{|c|}{ V } \\
\hline Organism B (receiver) & duced jump \\
\hline $\begin{array}{l}\text { ROTATE-RIGHT } \\
\ldots \\
\text { SEND-ALARM-MSG-LOCAL } \\
\text { MOVE } \\
\ldots \\
\text { ALARM-LABEL-HIGH } \\
\text { REPRO }\end{array}$ & $\begin{array}{l}\text { ROTATE-RIGHT } \\
\ldots \\
\text { SEND-ALARM-MSG-LOCAL } \\
\text { MOVE } \\
\ldots \\
\text { - ALARM-LABEL-HIGH } \\
\text { REPRO }\end{array}$ \\
\hline
\end{tabular}

\section{Figure 3. Example of the change in execution flow of organism $B$ when it receives a high- state alarm from organism $A$.}

Movement and Rotation. An organism can move to a neighboring cell by executing a MOVE instruction. The new location is determined by its facing. An organism can change its facing by executing a rotate instruction. The ROTATE-RIGHT and ROTATE-LEFT instructions allow an organism to rotate one cell to the right or left, respectively. We expanded the instruction set to include three additional types of rotation. The ROTATE-UNOCCUPIED-CELL and ROTATE-OCCUPIED-CELL instructions, respectively, rotate an organism clockwise until a cell is found that is unoccupied or occupied; if no such cell is found the organism's facing remains unchanged. The third new rotate instruction, ROTATE-TO-ATTACK-CELL, rotates an organism clockwise until a cell containing an attack event is found; if no attack is found the organism's facing remains unchanged. For example, in Figure 4 organism $A$ is initially facing to the left, but after executing a ROTATE-TO-ATTACK-CELL instruction it faces in the up direction.
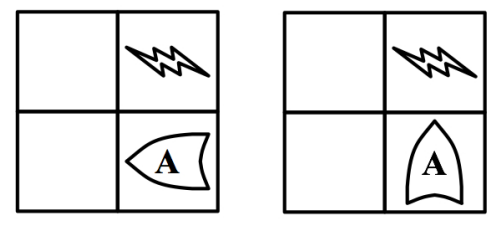

Figure 4. Example showing organism $A$ (represented by a boolean OR gate) before (left) and after (right) executing the ROTATE-TOATTACK-CELL instruction. The attack is represented by the lighting bolt.

Metabolic Rate. We also enabled an organism to have more direct control over its energy usage. In a prior study [9] we provided organisms with "sleep" instructions, and observed their evolution of circadian rhythms in response to periodic resource availability. Here, we added two instructions, DOUBLE-ENERGY-USAGE and HALVEENERGY-USAGE, to enable an organism to change its execution priority by either increasing or decreasing its metabolic rate. When an organism executes the DOUBLE-ENERGYUSAGE instructions, its metabolic rate is doubled, increasing the cycle speed of its virtual CPU. However, it will also pay double the energy cost per executed instruction. Moreover, if an organism increases its metabolic rate to a point where it can no longer pay an instruction's energy cost, then that organism dies. A third instruction, DEFAULT-ENERGYUSAGE, enables an organism to reset is energy usage to a default rate. In addition, upon replication both offspring organisms' energy usage return to the default level.

Dealing with attacks. The functionality to detect and quell an attack is available to an organism through special-purpose instructions. Specifically, we extended the Avida instruction set to include two conditional instructions that sense whether an organism is either in or facing a cell experiencing an attack. These two instructions, IF-CELL-UNDER-ATTACK and IF-FACED-CELLUNDER-ATTACK, cause the next instruction in an organism's genome to be skipped if an attack is not present in the interrogated cell. In addition to these two conditional in- 
structions, we also added two instructions that allow an organism to quell an attack. The KILL-ATTACK-IN-CELL and KILL-ATTACK-IN-FACED-CELL instructions, respectively, eliminate an attack in the organism's current cell, or the cell that it is facing. If an attack was present and mitigated, a 1 is written to the organism's BX register, otherwise a 0 is written. To more closely model resource requirements, these four attack-specific instructions, along with all movement, active messaging, and replication instructions, are assigned higher energy and virtual CPU costs than other instructions. The increased costs facilitate the evolution of efficient and effective solutions for finding and quelling attacks.

In the experiments presented in the following sections the rate of attacks during an attack period is randomly chosen to be either 0 or 5 attacks entering a deme per update. Every attack period lasts for 50 updates. Deme-level selection is used: a deme-level predicate is satisfied when a deme has successfully quelled $50 \%$ of all attacks that arrived during 5 consecutive attack periods. Once a deme has satisfied the deme-level predicate, it is replicated. At that point all of the deme's remaining energy is accumulated and divided equally among all cells in the offspring demes, minus a $5 \%$ deme replication decay. The transfer of remaining energy provides offspring of an energy efficient deme with a competitive advantage over less efficient demes. In addition, a previous study has shown that transferring energy in this manner can decrease the amount of time required to evolve cooperative behaviors [10].

\section{Experimental Results and Analysis}

We conducted a set of Avida experiments to investigate whether digital evolution could solve the problem of quelling attacks while conserving energy, and if so, what behaviors it might produce. All the runs used Avida populations containing up to 4900 organisms, arranged in 100 demes, each consisting of a $7 \times 7$ torus of cells. When a deme is initialized, each cell is given 10,000 energy units to be consumed by occupying organisms. A single seed organism is injected into the deme and begins to replicate, with mutations turned off (deme populations are homogeneous). If the population eventually satisfies the deme predicate, the deme will be replicated immediately. If a deme does not satisfy the predicate before it has experienced 500 updates, it will be replicated automatically because of its age. Upon deme replication, the germline may experience mutations. Specifically, there is a $0.75 \%$ probability that an instruction will mutated to a random instruction, and a $5 \%$ probability that an instruction will be inserted and removed.

An Evolved Solution. Execution of the Avida runs described above produced populations that dynamically adjusted their population in order to quell attacks while conserving energy. Figure 5 demonstrates this behavior for a particular population. The figure plots the mean fraction of cells within a deme containing an organism during periods of attack and during periods of "calm" (no attacks). As shown, very early in the run the population evolves the ability to keep the population near its capacity when attacks are present, and it maintains this behavior during the entire run. After approximately 40,000 updates, the population has evolved the ability to adaptively reduce its population (to between $30 \%$ to $60 \%$ of its maximum size) during calm periods.

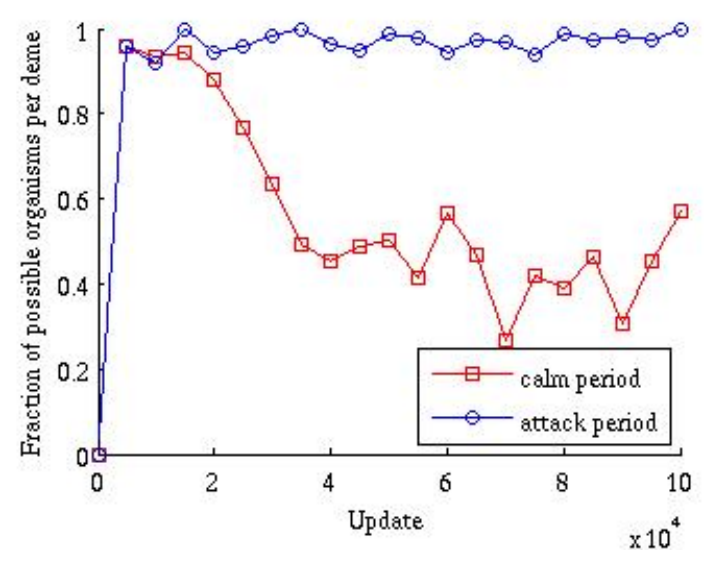

Figure 5. Mean fraction of total possible organisms within a deme when attacks are present and when they are not.

In addition to adaptively controlling the population, this particular population also achieves a mean attack mitigation rate of about a 90\%, as shown in Figure 6. Specifically, out of the 250 attacks occurring randomly during an attack period, about 225 are being quelled. We emphasize that the only selective pressure is the deme-level predicate requiring a 50\% mitigation rate. No evolutionary "building blocks" are used in these experiments, that is, we do not provide any reward for low-level behaviors from which the more complex cooperative behavior might emerge. Rather, the populations are entirely responsible for evolving their strategy.

Genome Analysis. In order to understand how digital evolution went about solving this problem, we analyzed the dominant genome present at the end of the run, shown in Figure 7. This genome contains two separate pieces of code, one that executes when attacks are present and the other when they are not. The genome initially attempts to quell ("kill") an attack in the cell that it is facing. The remainder of the execution of this genome depends on the success or failure of this attempt.

If no attack was killed, then the BX register will contain 0 and a low-state alarm will be sent to neighboring nodes. 


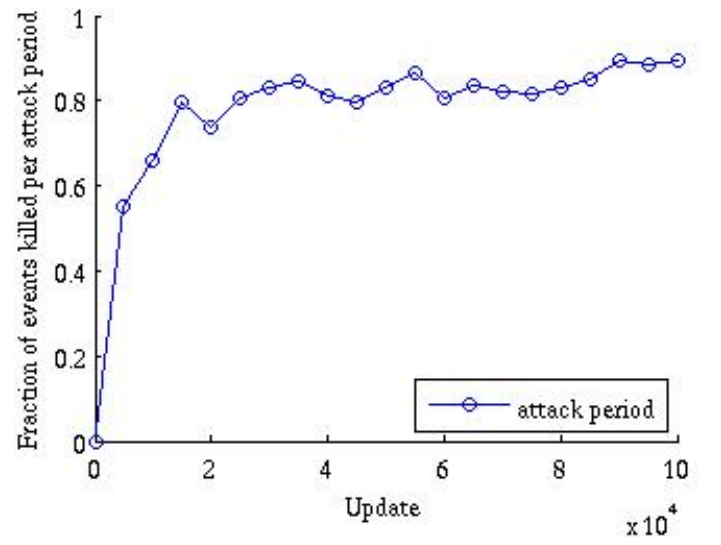

Figure 6. Average fraction of attacks quelled per attack period in a deme for a single run.

However, this alarm will have no effect on neighboring organisms in the deme because the genome does not contain any ALARM-LABEL-LOW instructions. Therefore the remainder of the code in Box 1 of Figure 7 will be executed at both the sender and its neighbors. This code moves the organism and then executes a SHIFT-R instruction on the BX register. The right shift causes the organism's BX register to either remain zero, or to become zero if an attack was previously killed. Then the organism enters into a low-energy (or "sleep") cycle, which costs the organism 1 energy unit and lasts for 30 virtual CPU cycles. Once the sleep cycle is over the organism replicates into the cell it is facing. Considering that demes are homogeneous, this method of replication allows the population of organism to remain low. Specifically, because a parent organism never changes its current facing, the parent and offspring organism will always remain in the same row, column, or diagonal. Therefore, when either of them replicates, the population can only grow only until that row, column or diagonal is full. After that point, all replications will replace existing organisms, causing the population size of the deme to remain constant.

On the other hand, if the attempt to kill an attack at the beginning of the genome was successful, then a high-state alarm is sent (since the value of BX is 1), and all organisms in the sender's neighborhood will jump to the top of Box 2 in Figure 7. If an organism is sleeping, it will remain sleeping, however its instruction pointer will be moved when it awakes in response to the most recently received alarm. The code within Box 2 can be divided into two parts: controlled spreading of alarm message, and racing to expand the population. The second and third lines in Box 2 control whether or not an organism that received an alarm message sends another alarm message. This piece of code will cause organisms that experience a high-state alarm message

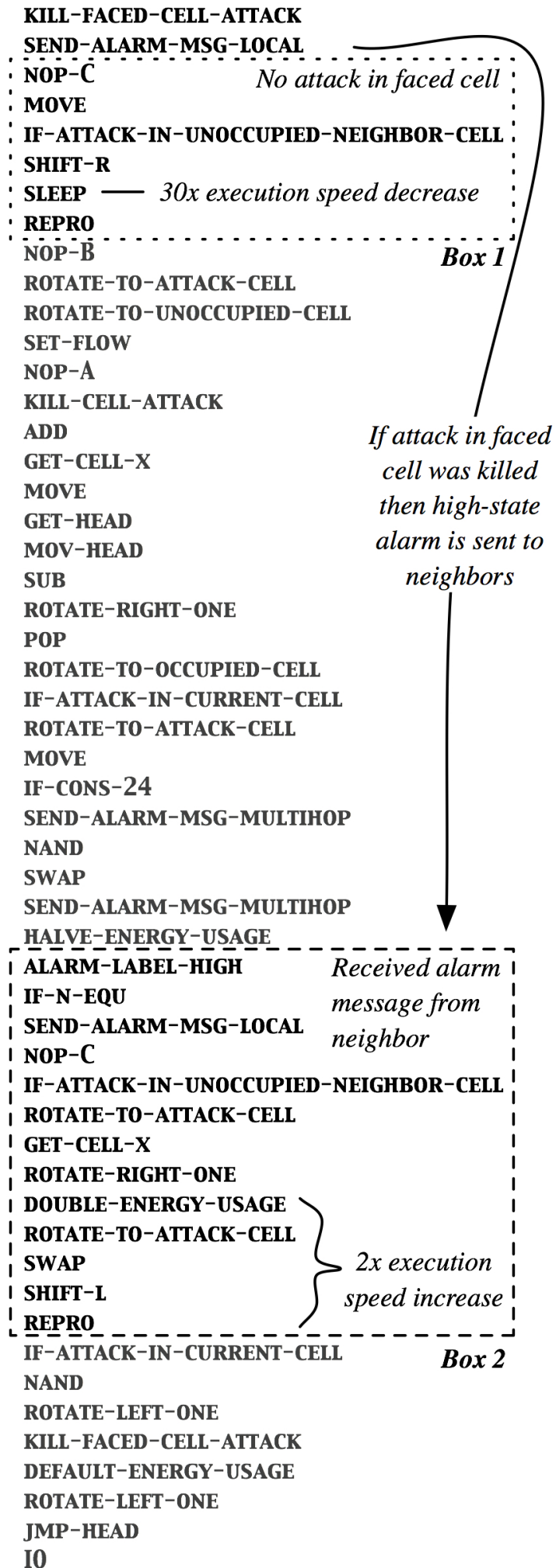

Figure 7. An evolved genome that executes differently depending on if a neighbor has sent a local high-state alarm message. 


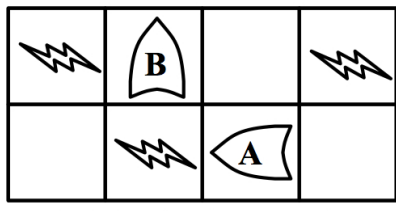

1. A begins to execute

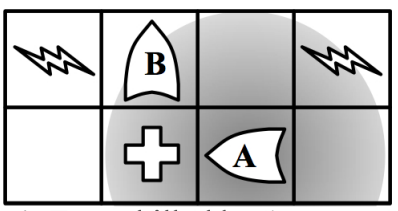

1. Event killed by $\mathbf{A}$

2. High alarm sent by $\mathbf{A}$

3. B's execution jumped

to ALARM-LABEL-HIGH (a)

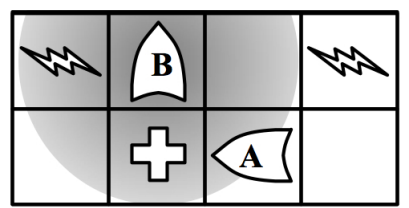

1. A's BX register set to zero by SHIFT-R

2. B sent high-state alarm

3. A's execution jumped

to ALARM-LABEL-HIGH

(c)

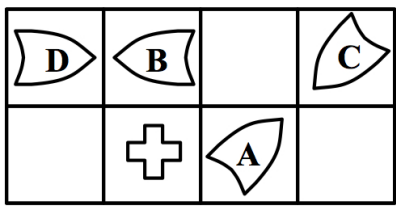

1. Alarm suppressed by $\mathbf{A}$

2. A \& B rotate to attacks, double execution rate, and replicate

3. Both attacks are killed

(d)

Figure 8. Sample population experiencing a period of attack. An organism is represented by a boolean OR gate where the point (or output) of the gate denotes the organism's facing. In addition, attacks, quelled attacks, and alarm messages are represented by lighting bolts, crosses, radially blended gray circle, respectively. Underlying each figure is a description of the individual executions represented.

to propagate the message unless the organism's BX and CX registers contain the same value. As discussed before, the contents of an organism's BX register after it exits a sleep cycle will be zero. Therefore an organism that entered a sleep cycle will not send an alarm message when it wakes up, so out-of-date alarms will not be propagated through the network. The second portion of code in Box 2 doubles the execution rate of the organism and rotates it to face an attack if one is present in a neighboring cell. Finally, the organism replicates. After replication, the parent organism, which is facing the offspring, will kill any event in the offspring's cell, a rather distinct evolved parental behavior.

Figure 8 is a graphical depiction of the execution of two organisms during an attack period scenario. In Figure 8(a) organism $A$ begins the execution of its genome, and organism $B$ is assumed not to be sleeping. After organism $A$ executes its first two instructions, the attack previously located in the bottom row has been quelled, $A$ has sent a high-state alarm, and $B$ 's instruction pointer has been jumped to the location of the ALARM-LABEL-HIGH instruction, as shown in Figure 8(b). After these instructions are executed, organism $A$ shifts its BX register to the right, causing its contents to be changed from a 1 to a 0 . At this point, organism $B$ sends a high-state alarm message which jumps $A$ 's instruction pointer to the location of the ALARM-LABEL-HIGH instruction. At this point, organism $A$ suppresses the sending of another alarm because its $\mathrm{BX}$ and $\mathrm{CX}$ registers contain the same value. Both organisms continue execution of instructions that cause them to rotate to face an attack, double their execution speed, and then replicate, as shown in Figure $8(\mathrm{~d})$. Immediately after replicating, both organisms $A$ and $B$ kill the attack in their respective offspring's cell. By doubling its execution speed, an organism will pay a higher energy cost per instruction, however, it increases the probability of quelling an attack in its offspring's cell, since the time between rotating to face the attack and attempting to kill it is cut in half.

Energy Conservation. When accounting for each instruction's user defined virtual CPU cycle cost, we calculate that the genome presented in Figure 7 can require as few a 9 cycles to execute during periods of attack and as many at 37 cycles to execute during calm periods. However, the lower bound on an organism's gestation time can only be achieved if a high-state alarm is received immediately after an organism replicates. This represents 4 -fold difference in individual organism gestation time depending on the current environment. Using these numbers to calculate the number of expected births during each period we predict a minimum of 1129 births during a calm period with $60 \%$ cell occupancy, and a maximum of 8167 births during an attack period. By examining Figure 9, we can see that the mean number of births during a calm period is slightly greater than expected. However, the mean number of births during attack periods is about half the expected total. This result suggests that organisms are replicating after executing about 18 instructions, which is 9 instructions more than required to execute the entire piece of code in the dashed box in Figure 7. We conclude that during attack periods, when high-state alarm messages are being sent, organisms are still conserving energy by sleeping when attacks are not present in their neighborhoods. On average, 1 in 5 organisms will sleep during a period of attack, conserving the energy in its cell.

Finally, we note that only 5 of the 20 runs achieved the level of success described above. Specifically, while most populations were similarly successful at quelling attacks, 


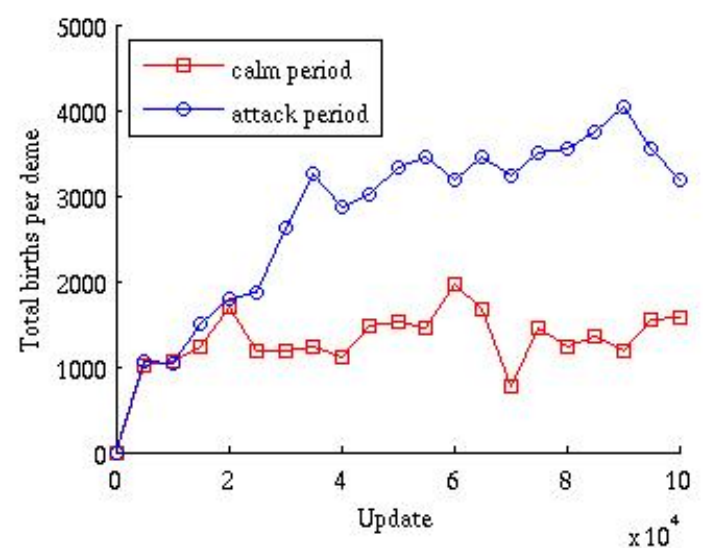

Figure 9. Mean number of births per deme in a single run.

only 5 were able to reduce the number of organisms significantly during calm periods. Of course, even a single successful population is sufficient for our purposes, as it can provide insight into the design of algorithms for agentbased distributed systems. However, from an evolutionary perspective this variation suggests that the selective pressures applied in this study might be improved, and in our ongoing studies we are exploring different pressures that may promote better energy efficiency and more effective self-regulation of population.

\section{Conclusions and Future Directions}

We have shown that it is possible to digitally evolve autonomous agents that are capable of adapting their population in response to attack fluctuations. Specifically, we described an evolved genome that self-regulates a population within a deme through the use of local active messaging. In addition, the genome adapts the execution speed of the host organism, depending on the current environmental circumstances. Finally, we showed that even during attack periods the evolved genome localizes the attack response, and conserves additional energy by putting about 1 in 5 organisms to sleep.

An additional benefit of leveraging DE for designing agent behavior is that every run produces many functionally equal genomes. As long as these solutions adequately solve the intended problem, they could be used to increase the heterogeneity of a system. Cyber attacks often rely on the target system's homogeneity by exploiting a vulnerability that is present throughout an entire system. By introducing new agents into a system periodically (effectively reconfiguring the system) the demographics of the system over an extended period of time would continue to change. There- fore an attack based on assumptions about the operation of the agents should be less likely to succeed.

In an agent-based distributed system, both individual lifetime and population size are important concerns for developers. Mismanagement of either of these two concerns can cause a disruption of a system's required QoS. We plan to extend this work into the design of real-world systems capable of self-regulating their populations and adapting the lifetime of their agents. Specifically, we intend to apply these results to the design of agent-based event/intrusion detection systems for use in wireless sensor networks. By drawing inspiration from natural systems and harnessing the evolutionary process which produced those systems, we hope to provide tools capable of handling the escalating complexity of distributed computing systems.

Further Information. Information on evolving adaptive and cooperative behavior can be found at http:// www. cse.msu.edu/thinktank. Papers on other applications of digital evolution and the Avida software are available at http://devolab.cse.msu.edu.

Acknowledgments. We would like to thank Charles Ofria, Dave Knoester and Brian Connelly for their contributions to this work. This study was supported in part by National Science Foundation grant CCF-0750787, CNS0751155, CCF-0820220 and by a Quality Fund Program grant from Michigan State University.

\section{References}

[1] A. Abraham and C. Grosan. Evolving intrusion detection systems. In N. Nedjah, A. Abraham, and L. de Macedo Mourelle, editors, Genetic Systems Programming: Theory and Experiences, volume 13 of Studies in Computational Intelligence, pages 57-80. Springer, Germany, 2006.

[2] C. Adami. Introduction to Artificial Life. Springer-Verlag New York, Inc., New York, NY, USA, 1998.

[3] C. Adami, C. A. Ofria, and T. C. Collier. Evolution of biological complexity. Proceedings of the National Academy of Sciences, 97(9):4463-4468, April 2000.

[4] J. Arabas, Z. Michalewicz, and J. J. Mulawka. GAVaPS a genetic algorithm with varying population size. In Proceedings of the International Conference on Evolutionary Computation, pages 73-78, 1994.

[5] M. Bakhouya and J. Gaber. Adaptive approach for the regulation of a mobile agent population in a distributed network. In Proceedings of the Fifth International Symposium on Parallel and Distributed Computing, pages 360-366, Washington, DC, USA, 2006.

[6] J. S. Balasubramaniyan, J. O. Garcia-Fernandez, D. Isacoff, E. Spafford, and D. Zamboni. An architecture for intrusion detection using autonomous agents. In Proceedings of the 14th Annual Computer Security Applications Conference, page 13, Washington, DC, USA, 1998. IEEE Computer Society. 
[7] B. Beckmann, P. K. McKinley, D. B. Knoester, and C. Ofria. Evolution of cooperative information gathering in selfreplicating digital organisms. In Proceedings of the First IEEE International Conference on Self-Adaptive and SelfOrganizing Systems (SASO), Boston, Massachusetts, July 2007.

[8] B. E. Beckmann, L. M. Grabowski, P. K. McKinley, and C. Ofria. Autonomic software development methodology based on Darwinian evolution (poster summary). In Proceedings of the 5th IEEE International Conference on Autonomic Computing, Chicago, Illinois, June 2008.

[9] B. E. Beckmann, P. K. McKinley, and C. Ofria. Evolution of an adaptive sleep response in digital organisms. In $A d$ vances in Artificial Life - Proceedings of the 9th European Conference on Artificial Life, volume 4648 of Lecture Notes in Computer Science, pages 233-242. Springer, 2007.

[10] B. E. Beckmann, P. K. McKinley, and C. Ofria. Selection for group-level efficiency leads to self-regulation of population size. In Proceedings of the 10th Annual Conference on Genetic and Evolutionary Computation, 2008.

[11] A. Bieszczad, T. White, and B. Pagurek. Mobile agents for network management. IEEE Communications Surveys, 1998.

[12] M. Burgess. Computer immunology. In Proceedings of the 12th Conference on Systems Administration, pages 283-298, Berkeley, CA, USA, 1998. USENIX Association.

[13] R. R. Cazangi, F. J. Von Zuben, and M. F. Figueiredo. Stigmergic Autonomous Navigation in Collective Robotics, volume 31 of Studies in Computational Intelligence, chapter 2. Springer Berlin / Heidelberg, 2006.

[14] S. Chow, C. O. Wilke, C. Ofria, R. E. Lenski, and C. Adami. Adaptive radiation from resource competition in digital organisms. Science, 305:84-86, 2004.

[15] M. Crosbie and E. H. Spafford. Defending a computer system using autonomous agents. Technical Report COAST TR 95-02, Department of Computer Sciences, 1995.

[16] H. Eskandari, C. D. Geiger, and G. B. Lamont. FastPGA: A dynamic population sizing approach for solving expensive multiobjective optimization problems. In Proceedings of the 4th International Conference on Evolutionary MultiCriterion Optimization, volume 4403 of Lecture Notes in Computer Science, pages 141-155. Springer, 2006.

[17] C. Fernandes and A. C. Rosa. Self-regulated population size in evolutionary algorithms. In PPSN, volume 4193 of Lecture Notes in Computer Science, pages 920-929. Springer, 2006.

[18] D. Floreano, S. Mitri, S. Magnenat, and L. Keller. Evolutionary conditions for the emergence of communication in robots. Current Biology, 17:514-519, March 2007.

[19] C.-L. Fok, G.-C. Roman, and C. Lu. Rapid development and flexible deployment of adaptive wireless sensor network applications. In Proceedings of the 25th IEEE International Conference on Distributed Computing Systems, pages 653662, Washington, DC, USA, 2005. IEEE Computer Society.

[20] S. Forrest, S. A. Hofmeyr, and A. Somayaji. Computer immunology. Communications of the ACM, 40(10):88-96, 1997.

[21] M. Glickman, J. Balthrop, and S. Forrest. A machine learning evaluation of an artificial immune system. Evolutionary Computation, 13(2):179-212, 2005.
[22] J. H. Holland. Adaptation in natural and artificial systems: An introductory analysis with applications to biology, control, and artificial intelligence. University of Michigan Press, 1975.

[23] C. A. Janeway, P. Travers, M. Walport, and M. J. Shlomchik. Immunobiology : the immune system in health and disease. Garland Publishing, New York, NY, USA, 5 edition, 2001.

[24] J. O. Kephart and D. M. Chess. The vision of autonomic computing. IEEE Computer, 36(1):41-50, 2003.

[25] D. B. Knoester, P. K. McKinley, B. Beckmann, and C. A. Ofria. Directed evolution of communication and cooperation in digital organisms. In Proceedings of the 9th European Conference on Artificial Life, Lisbon, Portugal, September 2007.

[26] D. B. Knoester, P. K. McKinley, and C. A. Ofria. Using group selection to evolve leadership in populations of selfreplicating digital organisms. In Proceedings of the Genetic and Evolutionary Computation Conference, July 2007.

[27] D. B. Knoester, P. K. McKinley, and C. A. Ofria. Cooperative network construction using digital germlines. In Proceedings of the ACM Genetic and Evolutionary Computation Conference, Atlanta, Georgia, July 2008.

[28] R. E. Lenski, C. Ofria, R. T. Pennock, and C. Adami. The evolutionary origin of complex features. Nature, 423:139$144,2003$.

[29] R. E. Lenski, C. A. Ofria, T. C. Collier, and C. Adami. Genome complexity, robustness and genetic interactions in digital organisms. Nature, 400:661-664, 1999.

[30] Q. Lv, P. Cao, E. Cohen, K. Li, and S. Shenker. Search and replication in unstructured peer-to-peer networks. In 16th ACM International Conference on Supercomputing, New York, USA, June 2002.

[31] P. K. McKinley, B. H. Cheng, C. Ofria, D. Knoester, B. E. Beckmann, and H. Goldsby. Harnessing digital evolution. IEEE Computer, 41(1):54-63, January 2008.

[32] C. Ofria and C. O. Wilke. Avida: A software platform for research in computational evolutionary biology. Artificial Life, 10:191-229, March 2004.

[33] T. von Eicken, D. E. Culler, S. C. Goldstein, and K. E. Schauser. Active messages: a mechanism for integrated communication and computation. In Proceedings of the 19th Annual International Symposium on Computer Architecture, pages 256-266, New York, NY, USA, 1992. ACM.

[34] T. White, B. Pagurek, and D. Deugo. Management of mobile agent systems using social insect metaphors. In Proceedings of the 21st IEEE Symposium on Reliable Distributed Systems, pages 410-415, 2002.

[35] C. O. Wilke, J. Wang, C. Ofria, C. Adami, and R. E. Lenski. Evolution of digital organisms at high mutation rate leads to survival of the flattest. Nature, 412:331-333, 2001.

[36] D. S. Wilson. Introduction: Multilevel selection theory comes of age. The American Naturalist, 150(S1-S4), July 1997. 